\section{0-159 DOES RADON CAUSE DISEASES OTHER THAN LUNG CANCER? RESULTS FROM THE GERMAN URANIUM MINER COHORT STUDY}

${ }^{1}$ Nora Fenske, Veronika Deffner, Maria Schnelzer, Michaela Kreuzer. ${ }^{1}$ Federal Office for Radiation Protection, Germany

\subsection{6/OEM-2021-EPI.119}

Introduction and Objectives Radon is an established risk factor for lung cancer. Less clear is whether radon causes other diseases than lung cancer. To further investigate such risks, updated mortality data from the German uranium miner cohort study (1946-2013) were analyzed.

Methods The cohort includes 58,974 men who were employed for at least 6 months between 1946 and 1989 at the former Wismut uranium mining company in Eastern Germany. Considered endpoints were mainly cancers other than lung cancer, circulatory diseases and non-malignant respiratory diseases. Exposure to radon and its progeny and external gamma radiation was retrospectively estimated through a comprehensive job-exposure matrix. Statistical modelling was performed by internal Poisson regression for grouped data with baseline stratification by age and calendar year. Excess relative risks (ERR) per 100 WLM (Working Level Month) and 95\% confidence intervals (CI) were estimated for cumulative 5-year lagged exposure to radon $($ mean $=280$, median $=33$, $\max =3,224$ WLM).

Results Within the follow-up period 1946-2013, a total of 5,122 cancer deaths other than lung cancer occurred. In this group, a statistically significant association was found (ERR/ $100 \mathrm{WLM}=0.014$; 95\% CI: 0.006, 0.021). Regarding individual cancer sites, the majority of risk estimates were positive (14 out of 18), and two of them reached borderline significance (colon and liver cancer). This holds true even after adjustment for external gamma radiation. No increased ERR/ 100 WLM was found for the groups of deaths from circulatory diseases $(n=10,665)$ and from non-malignant respiratory diseases excluding silicosis/other pneumoconiosis $(n=1,674)$.

Conclusion Our findings provide some evidence of an increased mortality risk for cancers other than lung cancer associated with radon. Chance, confounding by unconsidered risk factors and uncertainty in exposure assessment cannot be completely ruled out. If present at all, the radon-related risk for diseases and cancers other than lung cancer is substantially lower than that for lung cancer.

\section{0-174 MORTALITY IN A COHORT OF GERMAN UNDERGROUND URANIUM MINERS, 1960-2013}

${ }^{1}$ Veronika Deffner, Nora Fenske, Maria Schnelzer, Michaela Kreuzer. ${ }^{1}$ Federal Office for Radiation Protection, Germany

\subsection{6/OEM-2021-EPI.120}

Introduction and Objective Former German uranium miners had been exposed underground in the early years to high levels of radon and silica dust. The objective of these analyses is to compare mortality in the Wismut cohort with that of the male general population.

Methods A sub-cohort of 35,204 miners who worked at least 180 days between 1946 and 1989 underground, but never in processing or open pit mining was defined with observation period from 1960 to 2013. The underlying cause of death was available for $96.1 \%$ of the 18,510 deceased. For numerous causes of death, observed deaths (O) were compared with expected deaths (E) based on external mortality rates for the general male population by standardized mortality ratios $(\mathrm{SMR}=\mathrm{O} / \mathrm{E})$. Exposure to radon progeny and silica was retrospectively estimated by job-exposure matrices. SMR trends for different exposure levels were examined by the Poisson trend statistic.

Results A significant excess of lung cancer deaths was found $(\mathrm{O}=2,960 ; \mathrm{SMR}=2.36 ; 95 \% \mathrm{CI}: 2.28-2.45)$. Of the 27 considered cancer sites other than lung, SMR was statistically significantly increased for liver $(\mathrm{O}=175 ; \mathrm{SMR}=1.34 ; 95 \% \mathrm{CI}$ : $1.15-1.55)$ and stomach $(\mathrm{O}=504 ; \mathrm{SMR}=1.28 ; 95 \% \mathrm{CI}: 1.17$ 1.40) cancers. A significant excess mortality from non-malignant respiratory diseases was found $(\mathrm{O}=1,928 ; \mathrm{SMR}=1.86$; 95\% CI: 1.78-1.94), mainly related to mortality from silicosis/ other pneumoconiosis $(\mathrm{O}=941 ; \mathrm{SMR}=22.62 ; 95 \% \mathrm{CI}: 21.20$ 24.11) and influenza/pneumonia $(\mathrm{O}=294 ; \mathrm{SMR}=1.13 ; 95 \%$ CI: 1.01-1.27). Additionally, significant mortality excesses occurred for infectious diseases $(\mathrm{O}=157 ; \mathrm{SMR}=1.18 ; 95 \%$ CI: 1.01-1.38) and cerebrovascular diseases $(\mathrm{O}=1,335$; $\mathrm{SMR}=1.33 ; 95 \% \mathrm{CI}: 1.26-1.41)$. SMRs for lung and liver cancer significantly increased with cumulative radon exposure; SMRs for silicosis/other pneumoconiosis significantly increased with silica exposure.

Conclusion Underground miners of this cohort showed a clear excess mortality for lung cancer and silicosis/other pneumoconiosis even for those later hired with lower exposures. Further research is ongoing to investigate exposure-response-relationships via internal analyses in more detail.

\section{0-277 SOLAR UVR EXPOSURE AMONG OUTDOOR WORKERS IN ALBERTA: MEASUREMENTS AND PROTECTIVE BEHAVIOURS}

${ }^{1}$ Cheryl Peters, Ela Rydz, Andrew Harper, Brandon Leong, Victoria H Arrandale, Sunil Kalia, Thomas Tenkate, Lindsay Forsman-Phillips, D Linn Holness. ${ }^{1}$ University of Calgary, Canada

\subsection{6/OEM-2021-EPI.121}

Introduction Workplace solar UVR exposure has significant health and economic impacts. However, occupational exposure and sun protection behaviour data are scarcely available.

Objectives This study aimed to characterize outdoor workers' full-day solar UVR exposures and their determinants, as well as the prevalence and determinants of sun protection behaviours used at work and leisure.

Methods We collected personal dosimetry measurements over one week using calibrated, electronic UVR dosimeters, and outdoor workers' demographics, skin cancer risk factors, job information, and sun habits at work and leisure using selfcompleted questionnaires. Workers' mean daily solar UVR exposure (standard erythemal dose, SED), corrected for repeated measurements, was summarized, and determinants of exposure were assessed using marginal models. The frequency of specific protective behaviours at leisure and work was compared. Sun protection scores were calculated, and the determinants of these scores for both settings were modelled using multiple linear regression.

Results We recruited 179 workers and collected 883 full-day measurements. The mean dose among all workers was 1.9 SED (range: 0.03-16.6). Nearly half of all measurements exceeded the recommended international limit (1.3 SED). Landscape and maintenance workers (2.6 SED), and trade and 